\title{
Transient Cortical Blindness Following Vertebral Angiography: A Case Report
}

\author{
Lai Wan Lo, MD, Ho Fung Chan, MD, Ka Fai Ma, MD, \\ Lik Fai Cheng, MD, Tony KT Chan, MD
}

Transient cortical blindness (TCB) is a rare but well-known complication of cerebral angiography. Its pathophysiology remains uncertain. We would like to report a case of TCB in a patient during a follow up vertebral angiogram for post-coil embolization of left posterior inferior cerebellar artery aneurysm. Patient's vision was resumed spontaneously within 24 hours after angiography, with no residual neurological deficit in subsequent clinical follow up. Multi-modality imaging evaluation including vertebral angiography, brain CT and MRI performed on same day are presented.

Key Words : Transient Cortical Blindness; Cerebral Angiography; TCB

Transient cortical blindness (TCB) is a rare but wellknown complication of cerebral angiography. The incidence was reported to be $0.3-1 \%$ of cerebral angiography [1]. With newer contrast agents, the incidence of $0.2 \%$ was reported recently [2]. Its pathophysiology remains uncertain. Patients complained of cortical blindness within minutes of contrast injection and usually resolved within few days [1]. It is reported to have a higher incidence for vertebral angiography [3]. We would like to report a case of TCB following vertebral angiography with multimodality imaging performed.

All authors: Department of Radiology, Princess Margaret Hospital, Hong Kong

Received August 29, 2014;

accepted after revision February 23, 2015.

Correspondence to: Lai Wan Lo (MBBS, FRCR), Department of Radiology, LG1, Block B, Princess Margaret Hospital, Hong Kong Tel. 852-98340586

E-mail: wanllw@hotmail.com

This is an Open Access article distributed under the terms of the Creative Commons Attribution Non-Commercial License (http://creativecommons.org/licenses/by-nc/3.0) which permits unrestricted non-commercial use, distribution, and reproduction in any medium, provided the original work is properly cited.

\section{CASE REPORTS}

A 57-years-old male with good past health was presented to emergency department of our hospital on 2nd June 2013, complained of sudden onset of severe headache and vomiting without history of head injury. Patient was all along conscious with GCS 15/15 and no focal neurological deficit was elicited on physical examination. Urgent non-contrast CT showed diffuse acute subarachnoid haemorrhage (SAH). Diagnostic cerebral angiograms were performed on the next day. A $0.7 \mathrm{~cm}$ narrow-neck aneurysm was identified over the origin of left posterior inferior cerebellar artery (PICA). Coil embolization of the left PICA aneurysm was performed one day later. Small residual neck was left in order to preserve origin of left PICA. No immediate complication was noted. Patient showed good recovery with no vasospasm and was discharged on day 14 postSAH.

Elective follow-up left vertebral angiogram was performed 4 months later with right femoral approach and selective catheterization of left vertebral artery using 5Fr headhunter type catheter. The contrast agent used was Omnipaque $300 \mathrm{mg} / \mathrm{ml}$ and $20 \mathrm{ml}$ of contrast 
was used in total. Patient then developed bilateral blindness immediately after angiography. Patient remained normotensive during the angiography. Review of angiograms showed no sign of vascular occlusion or spasm in the vertebrobasilar territory (Fig. 1).

Urgent non-contrast brain CT was performed within 2 hours after angiography and showed no evidence of acute infarction over bilateral parieto-occipital cortex (Fig. 2). Brain MRI was arranged within 3 hours after angiography and demonstrated no abnormality on T1weighted, T2-weighted, and post-gadolinium T1weighted images. Post-gadolinium FLAIR MR images showed abnormal high signal over sulcal spaces of bilateral parieto-occipital lobes (Fig. 3). No restricted diffusion was seen on diffusion weighted images and apparent diffusion coefficient (Fig. 4). Assessment by an ophthalmologist revealed no local cause of sudden visual loss. Short course of steroid was given. Patient's vision was normalized within 24 hours after vertebral angiography. Patient was discharged one week later with no residual neurological deficit.

\section{DISCUSSION}

TCB is a rare but dramatic complication of cerebral angiogram. More than 600 cerebral angiograms were performed in our hospital over the past 5 years and this is our first case of TCB following cerebral angiogram $(0.16 \%)$.

The exact mechanism of TCB following cerebral angiography is unknown and there are two main hypotheses. The first hypothesis is that it is a result of the neurotoxic effect of contrast agent causing osmotic disruption of blood-brain barrier [4]. This hypothesis is supported by the different sympathetic innervation of posterior circulation, which is more susceptible to such injury [5]. The contrast agent used in our reported case was Omnipaque, a non-ionic contrast agent. When used without dilution, it is hyperosmotic to blood, contributing to disruption of blood-brain barrier. Reported MRI findings of blood-brain barrier disruption The second hypothesis is that TCB is the result of posterior reversible leukoencephalopathy syndrome (PRES) [6], which is due to failure of cerebrovascular autoregula-

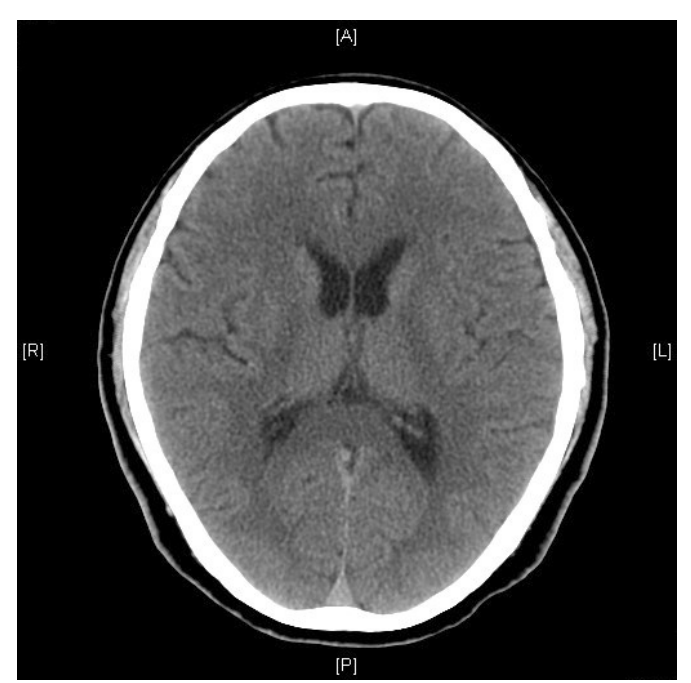

Fig. 2. Brain CT obtained 2 hours after angiography shows no evidence of acute infarction or intracranial hemorrhage.

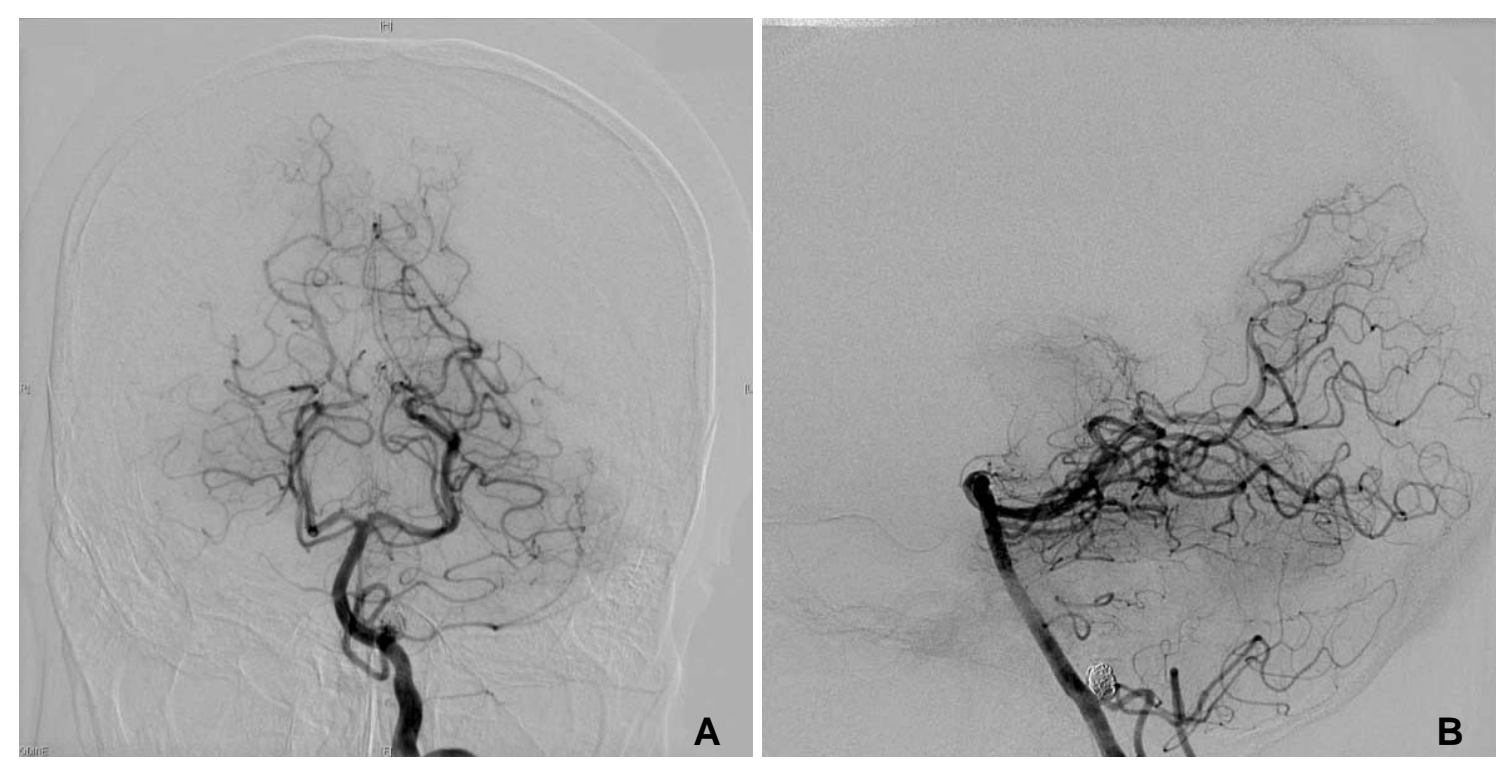

Fig. 1. Anteroposterior (A) and lateral (B) vertebral angiograms show no sign of vascular occlusion or spasm in the vertebrobasilar territory. Note the coiled aneurysm in the origin site of left posteroinferior cerebellar artery. 

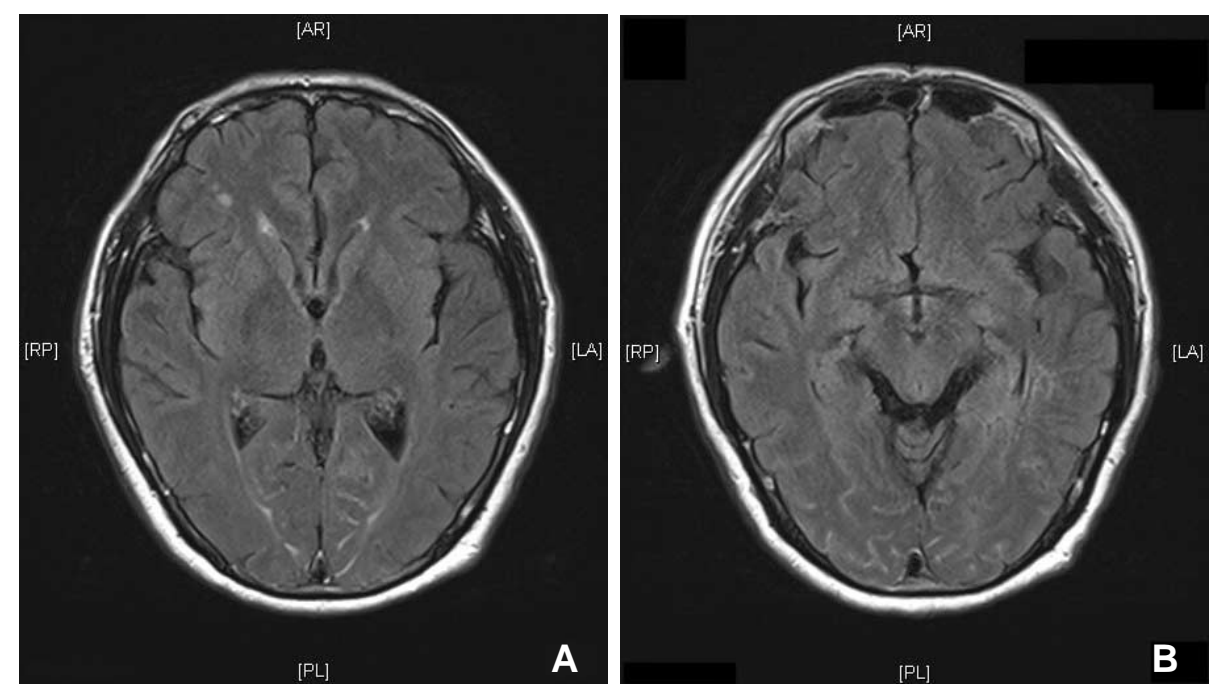

Fig. 3. Post-contrast FLAIR images (A, B) obtained 3 hours after angiography show high signal intensity lesions in the sulcal spaces of bilateral parieto-occipital lobes.

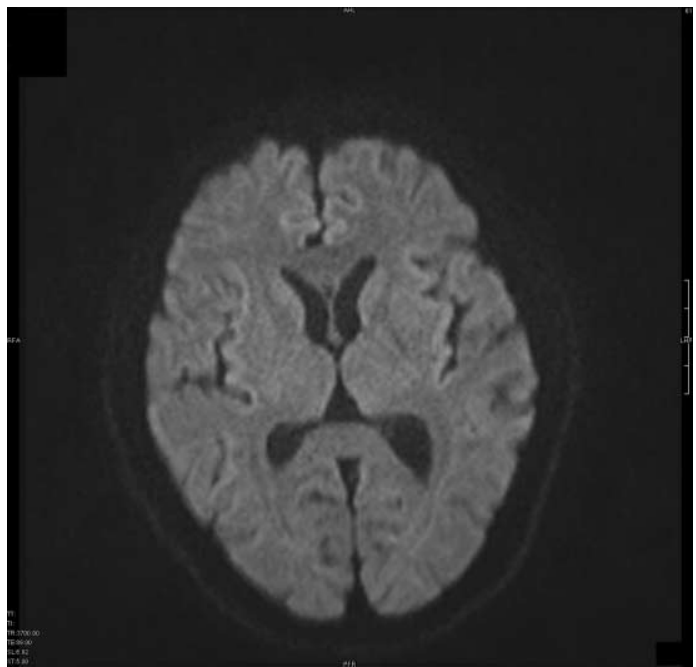

Fig. 4. Diffusion image obtained 3 hours after angiography shows no diffusion restricted lesions.

tion, leading to perivascular edema. Typical MRI features of PRES is subcortical distribution of high signal in FLAIR and DWI in parieto-occipital regions [7]. In our case, abnormal high signal intensity was noted in the sulcal spaces of bilateral parieto-occipital lobes on post-gadolinium FLAIR images, not in the subcortical white matter. No abnormal signal was detected on T1, T2 and post-gadolinium T1-weighted images. To our knowledge, these findings have not been described in patients with TCB previously in the literature. This finding may support the hypothesis of blood-brain barrier disruption by contrast neurotoxicity in patients with TCB. Subarachnoid high signal intensities on FLAIR images can be related to blood and exudates with high protein content. Post-gadolinium FLAIR has been suggested to be more sensitive for detecting abnormal signal intensity in subarachnoid spaces than post-gadolinium T1-weighted sequence [8]. In the case of blood-brain barrier dysfunction, leakage of gadolinium into extracellular space may shorten the T1 value causing FLAIR high signal intensity in the subarachnoid spaces.

In the clinical context of sudden blindness after cerebral angiography, one should differentiate TCB from embolic infarction as a complication of angiography. Bilateral blindness is more likely to be related to TCB rather than embolism affecting both occipital lobes which has been reported after cardiac catheterization though this is very rare [9]. The use of MRI is helpful in this context as there is no restricted diffusion of the affected occipital lobes in case of TCB as in our case, while there will be restricted diffusion in cases of acute infarction related to cerebral embolism.

The treatment of these two conditions are different as more active thrombolytic therapy may be needed in case of ischemic infarct, while for TCB no treatment is needed because it is self-limiting and reversible. In fact, no treatment has been proven to be effective to improve the natural history of TCB following cerebral angiography $[6,10]$. It usually lasts from a few hours to a few days. Patients should be reassured that symptoms will resolve spontaneously within few days.

In summary, we reported a case of TCB following vertebral angiography, a rare but dramatic complication of cerebral angiography. With our imaging finding of sulcal high signal intensity on post-gadolinium FLAIR images, the mechanism is more likely related to neurotoxicity of contrast agent injected during angiography with transient disruption of blood-brain barrier. Radiologists and clinicians should be aware of this 


\section{Lai Wan Lo, et al.}

complication. When it occurs, one should reassure the patient about the excellent prognosis of this condition.

\section{References}

1. Horwitz NH, Wener L. Temporary cortical blindness following angiography. J Neurosurg 1974;40:583-586

2. Till V, Koprivsek K, Stojanovic S, Avramov P, Vulekovic P. Transient cortical blindness following vertebral angiography in a young adult with cerebellar haemangioblastoma. Pediatr Radiol 2009;39:1223-1226

3. Wishart DL. Complications in vertebral angiography as compared to non-vertebral cerebral angiography in 447 studies. Am J Roentgenol Radium Ther Nucl Med 1971;113:527-537

4. Alsarraf R, Carey J, Sires BS, Pinczower E. Angiography contrast-induced transient cortical blindness. Am J Otolayngology 1999:130-132

5. Mentzel HJ, Blume J, Malich A, Fitzek C, Reichenbach JR, Kaiser WA. Cortical blindness after contrast-enhanced CT: complication in a patient with diabetes insipidus. AJNR Am J
Neuroradiol 2003;24:1114-1116

6. Saigal G, Bhatia R, Bhatia S, Wakhloo AK. MR findings of cortical blindness following cerebral angiography: is this entity related to posterior reversible leukoencephalopathy? AJNR Am J Neuroradiol 2004;25:252-256

7. Fugate JE, Claassen DO, Cloft HJ, Kallmes DF, Kozak OS, Rabinstein AA. Posterior reversible encephalopathy syndrome: associated clinical and radiological findings. Mayo Clinic Proc 2010;85:427-432

8. Mathews VP, Caldemeyer KS, Lowe MJ, Greenspan SL, Weber DM, Ulmer JL. Brain: gadolinium-enhanced fast fluid-attenuated inversion-recovery MR imaging. Radiology 1999;211:257-263.

9. Papaconstantinou D, Georgalas I, Diagourtas A, Georgopoulos G, Ladas I, Gotsis S. Cortical blindness due to bilateral embolism: a rare complication of cardiac catheterization. Clin Exp Optom 2010;93:366-367

10. Gellen B, Remp T, Mayer T, Milz P, Franz WM. Cortical blindness: a rare but dramatic complication following coronary angiography. Cardiology 2003;99:57-59 